\title{
Identification of Rice Chromosome Segment Substitution Line Z322-1-10 and Mapping QTLs for Agronomic Traits from the $F_{3}$ Population
}

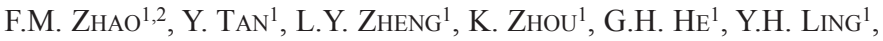 \\ L.H. ZHANG ${ }^{2}$ and S.Z. XU ${ }^{2 *}$ \\ ${ }^{1}$ Laboratory of Application and Safety Control of Genetically Modified Crops, Rice Research Institute, \\ Southwest University, Chongqing, China, 400716 \\ ${ }^{2}$ Department of Botany and Plant Sciences, University of California, Riverside, CA, 92521, USA
}

(Received 19 February 2016; Accepted 20 April 2016;

Communicated by J. Zhang)

\begin{abstract}
Chromosome segment substitution lines (CSSLs) are powerful tools to combine naturally occurring genetic variants with favorable alleles in the same genetic backgrounds of elite cultivars. An elite CSSL Z322-1-10 was identified from advanced backcrosses between a japonica cultivar Nipponbare and an elite indica restorer Xihui 18 by SSR marker-assisted selection (MAS). The Z322-1-10 line carries five substitution segments distributed on chromosomes 1, 2, 5, 6 and 10 with an average length of $4.80 \mathrm{Mb}$. Spikilets per panicle, 1000-grain weight, grain length in the Z322-1-10 line are significantly higher than those in Nipponbare. Quantitative trait loci (QTLs) were identified and mapped for nine agronomic traits in an $\mathrm{F}_{3}$ population derived from the cross between Nipponbare and Z322-1-10 using the restricted maximum likelihood (REML) method in the HPMIXED procedure of SAS. We detected 13 QTLs whose effect ranging from $2.45 \%$ to $44.17 \%$ in terms of phenotypic variance explained. Of the 13 loci detected, three are major QTL ( $q$ GL1, qGW5-1 and $q R L W 5-1$ ) and they explain $34.68 \%, 44.17 \%$ and $33.05 \%$ of the phenotypic variance. The $q G L 1$ locus controls grain length with a typical Mendelian dominance inheritance of 3:1 ratio for long grain to short grain. The already cloned QTL $q G W 5-1$ is linked with a minor QTL for grain width $q G W 5-2(13.01 \%)$ in the same substitution segment. Similarly, the previously reported $q R L W 5-1$ is also linked with a minor QTL $q R L W 5-2$. Not only the study is important for fine mapping and cloning of the gene $\mathrm{q} G L 1$, but also has a great potential for molecular breeding.
\end{abstract}

Keywords: chromosome segment substitution line, QTL mapping, rice, yield, yield component trait

\section{Introduction}

Rice (Oryza sativa L.) serves as a main staple food for half of the world population. High yield has always been the target of rice breeding. However, yield-related traits are governed by many quantitative trait loci (QTLs) and easily influenced by genetic backgrounds

\footnotetext{
*Corresponding author; E-mail: shizhong.xu@ucr.edu
} 
and environments (Liao et al. 2001). To date, large numbers of QTLs for yield and its components have been reported (Xing and Zhang 2010) and some major QTLs have been cloned, such as GS3 (Fan et al. 2006), GL3. 1 (Peng et al. 2012), GW2 (Song et al. 2007), $q G W 8$ (Wang et al. 2012), GW5 (Weng et al. 2008) and Gnla (Ashikari et al. 2005). These studies facilitated a better understanding of the regulation mechanism of grain yield in rice. However, insufficient numbers of effective favorable alleles may impede the application of these QTLs in rice breeding programs (Zheng et al. 2011).

Chromosome segment substitution lines (CSSLs) can be used both for QTL mapping and for new cultivar development as female lines due to the unique feature of uniform genetic background (Furuta et al. 2014). Therefore, development of CSSLs is a useful step to improve breeding programs (Zamir 2001). Some CSSLs have been developed and used in isolation of alleles of rice target QTL (Ebitani et al. 2005; Wan et al. 2006; Yang et al. 2015). However, the QTLs were derived from naturally occurring variation, and favorite alleles are often separated in different germplasms. The use of a wider range of genetic variation is important (Takai et al. 2007) but the limited number of crossovers do not allow us to explore all naturally occurring genetic variants in rice. Therefore, novel CSSLs need to be developed.

In this study, we report a novel elite CSSL, named Z322-1-10, which was developed through advanced backcrosses between the recipient cultivar Nipponbare (a japonica rice cultivar) and the donor Xihui 18 (an elite indica rice restorer) by SSR markers MAS. We will describe how the CSSL Z322-1-10 was developed, how to perform QTL mapping using an $\mathrm{F}_{3}$ population derived from this line and the statistical method used to perform data analysis.

\section{Materials and Methods}

\section{Materials}

An elite CSSL named Z322-1-10 was used as the material in the study. Z322-1-10 was derived from advanced backcrosses between Nipponbare as the recipient parent and Xihui 18 as the donor by SSR markers MAS. The Z322-1-10 line in $\mathrm{BC}_{3} \mathrm{~F}_{4}$ is stable in inheritance. Nipponbare was an elite japonica rice cultivar which has been fully sequenced. The Xihui 18 is an elite indica rice restorer bred by our laboratory.

The material used for QTL analysis was the $\mathrm{F}_{3}$ population derived from this cross with 150 individuals. The $\mathrm{F}_{3}$ seeds were collected from $5 \mathrm{~F}_{2}$ individual plants with long grains derived from the cross of Nipponbare and Z322-1-10 in Hainan Province of China in 2013. All the $\mathrm{F}_{3}$ seeds and its parents (Nipponbare and Z322-1-10) were first pre-germinated by soaking in water at $25{ }^{\circ} \mathrm{C}$ for $48 \mathrm{~h}$, and raised in the greenhouse at Southwest University, Chongqing, China, in March of 2014. Thirty seedlings of Z322-1-10 and 30 seedlings of Nipponbare along with 150 seedlings of the $F_{3}$ population were transplanted in each plot of the experiment field 40 days after sowing with a spacing of $16.67 \mathrm{~cm}$ between hills and $26.67 \mathrm{~cm}$ between rows, with conventional field cultivation management. 


\section{Development of Z322-1-10}

The Z322-1-10 line was developed by crossing Nipponbare with Xihui18. The resulting $\mathrm{F}_{1}$ hybrid was backcrossed with Nipponbare to produce $7 \mathrm{BC}_{1} \mathrm{~F}_{1}$ followed by successive backcrossing to Nipponbare to obtain $53 \mathrm{BC}_{3} \mathrm{~F}_{1}$ progenies. A total of 530 plants from the 53 lines were genotyped with 182 polymorphism SSR markers evenly distributed among all 12 chromosomes of the rice genome. A line named Z205-1-2 with nine substitution segments carrying 21 markers, including 10 heterozygous markers and 11 homozygous ones was found possessing excellent panicle phenotype. Eventually, 20 plants from each advanced generations of Z205-1-2 were used to select target segments with both marker and phenotype selection. Finally, a stable CSSL named Z322-1-10 in $\mathrm{BC}_{3} \mathrm{~F}_{4}$ was identified. The line carried five substitution segments. The estimated length of the substitution segments was calculated using the method proposed by Paterson et al. (1991). The chromosome map was generated using the Mapchart 2.2 software package.

\section{Measurement of agronomic traits}

Heading date, plant height and leaf morphology between Z322-1-10 and Nipponbare are very similar and these traits are not of interest in this study. We measured the yield and yield related trait along with grain shape traits in the study. At maturity, 10 plants at the

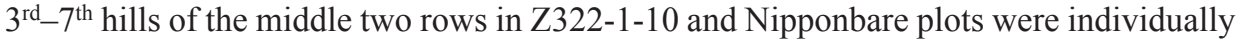
harvested and measured for grain length, grain width, ratio of length to width, 1000-grain weight, panicle number per plant, spikelets per panicle, grain number per panicle, and seed-setting ratio. $t$-tests were conducted between Z322-1-10 and Nipponbare for these traits. The same traits mentioned above were also measured for the $150 \mathrm{~F}_{3}$ individuals for QTL mapping. Simple statistical analysis such as $t$-test for the traits between Nipponbare and Z322-1-10 and the skewness and kurtosis in the $F_{3}$ population were conducted with intrinsic statistical functions within Excel 2003.

\section{Extraction of rice DNA}

Leaves of $150 \mathrm{~F}_{3}$ individuals and their plants were sampled to extract DNA at $20 \mathrm{~d}$ after transplanting. DNA extraction was conducted using the CTAB method of McCouch et al (1988) with a slight modification.

\section{PCR amplification and QTL mapping}

The total reaction volume of PCR was $12.5 \mu \mathrm{L}$, including $1.25 \mu \mathrm{L}$ of $10 \times \mathrm{PCR}$ buffer, 0.65 $\mu \mathrm{L}$ of $25 \mathrm{mmol} \mathrm{L}^{-1} \mathrm{MgCl}_{2}, 0.5 \mu \mathrm{L}$ of $2.5 \mathrm{mmol} \mathrm{L}^{-1} \mathrm{dNTPs}, 8.0 \mu \mathrm{L}$ of $\mathrm{ddH}_{2} \mathrm{O}, 1.0 \mu \mathrm{L}$ of

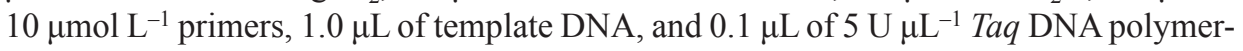
ase. After heating the PCR reaction to $94{ }^{\circ} \mathrm{C}$ and holding it for $3 \mathrm{~min}$ to complete the process of initial denaturation, the cycle at $94{ }^{\circ} \mathrm{C}$ was repeated for $20 \mathrm{~s}, 56^{\circ} \mathrm{C}$ for $20 \mathrm{~s}$, and $72{ }^{\circ} \mathrm{C}$ for $40 \mathrm{~s}, 35$ times. Finally, the extension step at $72{ }^{\circ} \mathrm{C}$ was undertaken for 7 
min. The PCR products were observed after the treatments of $10 \%$ native-PAGE and rapid silver dying (Panaud et al. 1996). The lanes of Nipponbare were marked by "-1", those of the heterozygotes were marked by " 0 ", and the lanes of Xihui18 were marked by " 1 ". Missing values were replaced by "." Lanes of each marker located on the substitution segments, together with the phenotypic values of each individual of the $\mathrm{F}_{3}$ population were used to identify the putative QTLs using the restricted maximum likelihood (REML) method implemented in the HPMIXED procedure in SAS (SAS Institute Inc. 2009). The $p$-value of 0.05 was used as the threshold to decide whether a QTL is linked with these markers in the substitution segments. Detected QTLs were named by a letter q followed by abbreviation of the trait-chromosome-serial number.

\section{Results}

\section{Identification of the substitution segments}

Based on the development of the Z322-1-10 line, three plants of Z322-1-10 were used to validate the target segments with all 11 markers located on the substitution segments and detect the residual segments with 36 other markers on the 12 chromosomes. The results showed that Z322-1-10 is homozygous with no residual segments being detected. The Z322-1-10 carries five chromosome substitution segments with a total length of $24.01 \mathrm{Mb}$ and an average length of $4.80 \mathrm{Mb}$. The five segments are distributed on chromosomes 1 , 2, 5, 6 and 10. The substitution segments on chromosomes 1 and 2 are short arm-RM3426 -RM1167 and RM6834-RM1920-RM112, respectively, whose estimated lengths were $2.40 \mathrm{Mb}$ and $4.80 \mathrm{Mb}$. The substitution intervals on chromosomes 5, 6 and 10 are $\mathrm{nS}$ SR505-RM289, RM7412-RM494 and RM7020-RM6673, respectively, and corresponding lengths are $6.97 \mathrm{Mb}, 3.50 \mathrm{Mb}$ and 6.34 Mb (Fig. 1).

\section{Phenotypic analysis of Z322-1-10}

Compared to Nipponbare, panicle length, number of spikelets per panicle and 1000-grain weight in Z322-1-10 are significantly increased by $1.96 \mathrm{~cm}, 42.7$ and 2.44 g, respectively, for the three traits. The seed setting ratio of Z322-1-10 (78.79\%) is significantly lower than that of Nipponbare (89.97). No differences were observed for panicle number per plant and grain number per panicle between CSSL Z322-1-10 and Nipponbare (Table 1). Grain length and ratio of grain length to grain width in Z322-1-10 are significantly higher than those in Nipponbare. Whereas, the grain width of Z322-1-10 is narrower than that of Nipponbare (Table 1), Therefore, Z322-1-10 has a high potential in QTL pyramiding because of these desirable traits.

\section{Frequency distributions of traits in the $F_{3}$ population}

To understand the genetic behaver of QTLs responsible for the phenotypic variation in Z322-1-10, frequency distributions of traits in the $F_{3}$ population are displayed. The values 

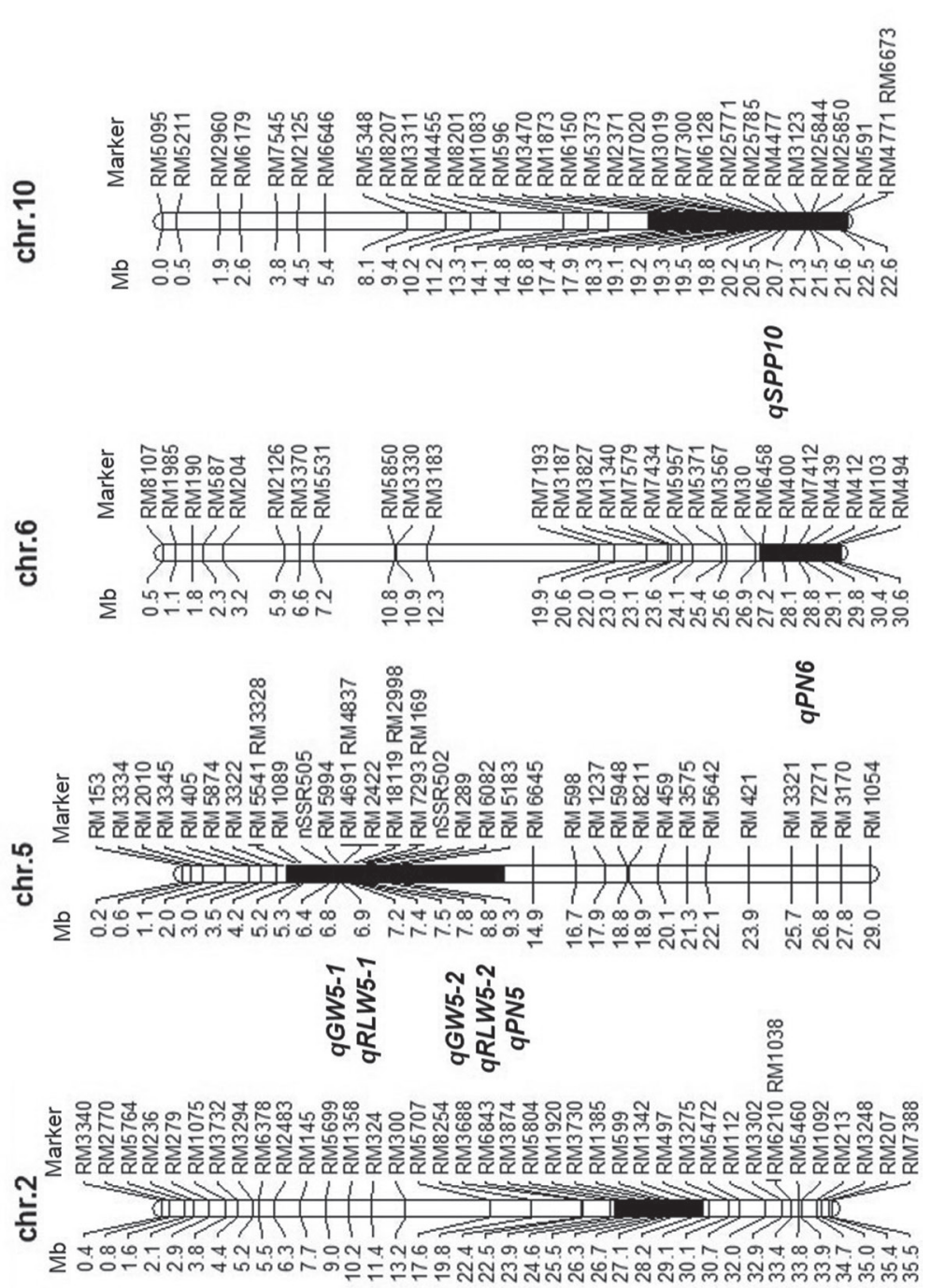

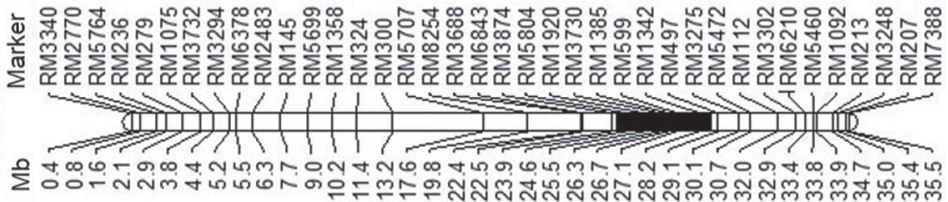
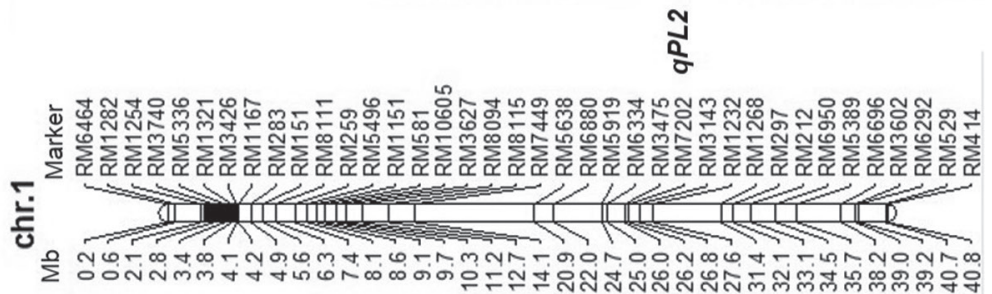
Table 1. Descriptive statistics of 10 important agronomic traits of Z322-1-10, Nipponbare and their $\mathrm{F}_{3}$ population

\begin{tabular}{|l|c|c|c|c|c|c|}
\hline \multirow{2}{*}{ Traits } & \multicolumn{2}{|c|}{ Parent } & \multicolumn{3}{c|}{$F_{3}$ population } \\
\cline { 2 - 7 } & Nipponbare & Z322-1-10 & mean \pm SD & Range & Skew & Kurt \\
\hline PL (cm) & $24.36 \pm 1.59^{\mathrm{a}}$ & $26.32 \pm 1.78^{\mathrm{b}}$ & $26.53 \pm 1.94$ & $21.7-32$ & 0.07 & -0.14 \\
\hline PN & $11.60 \pm 2.29^{\mathrm{a}}$ & $9.70 \pm 1.79^{\mathrm{a}}$ & $11.15 \pm 3.11$ & $4-27$ & 1.29 & 4.39 \\
\hline SPP & $141.30 \pm 12.92^{\mathrm{a}}$ & $184.00 \pm 24.94^{\mathrm{b}}$ & $186.42 \pm 41.15$ & $95-327$ & 0.53 & 0.99 \\
\hline GPP & $127.20 \pm 13.11^{\mathrm{a}}$ & $144.40 \pm 23.48^{\mathrm{a}}$ & $127.35 \pm 46.49$ & $0-237$ & -0.53 & 0.42 \\
\hline SSR (\%) & $89.97 \pm 2.99^{\mathrm{a}}$ & $78.79 \pm 10.77^{\mathrm{b}}$ & $69.22 \pm 22.64$ & $0-96.64$ & -1.18 & 0.85 \\
\hline GWT (g) & $21.33 \pm 0.75^{\mathrm{a}}$ & $23.77 \pm 1.02^{\mathrm{b}}$ & $22.30 \pm 2.89$ & $14.2-27$ & -3.29 & 1.37 \\
\hline GL (mm) & $7.00 \pm 0.05^{\mathrm{a}}$ & $8.41 \pm 0.23^{\mathrm{b}}$ & $7.87 \pm 0.40$ & $7.00-8.81$ & 0.04 & -0.64 \\
\hline GW (mm) & $3.17 \pm 0.03^{\mathrm{a}}$ & $3.06 \pm 0.02^{\mathrm{b}}$ & $3.01 \pm 0.17$ & $2.6-3.8$ & 0.98 & 2.78 \\
\hline RLW & $2.21 \pm 0.02^{\mathrm{a}}$ & $2.77 \pm 0.03^{\mathrm{b}}$ & $2.61 \pm 0.21$ & $2.04-3.15$ & 0.03 & -0.03 \\
\hline
\end{tabular}

Note: number followed by ${ }^{(\mathrm{a})}$ and ${ }^{(\mathrm{b})}$ indicate significant difference at $\mathrm{P}<0.05 ; \mathrm{PL}$ - panicle length; $\mathrm{PN}$ - panicle number; $\mathrm{SPP}$ - number of spikelets per panicle; GPP - grains per panicle; SSR - seed setting rate; GWT - 1000-grain weight; GL - grain length; GW - grain width; RLW - ratio of length to width.

of skewness and kurtosis are important characters to evaluate for the assumed normal distributions. As shown in Table 1 and Fig. S1*, the skewness values of most traits are close to 0 and the kurtosis values are mostly positive, suggesting that the distribution of these traits except grain length and seed setting ratio are continuous and approximately normal. These results indicate that most traits in Z322-1-10 are controlled by multiple QTLs.

Among all the traits, only grain length shows a bimodal distribution (Fig. S1). The peak value of short grain length is distributed in the range of 7.00-7.53 $\mathrm{mm}$ with the Nipponbare value at about $7.00 \mathrm{~mm}$. The peak values of long grain length ranges from 7.54 to $8.81 \mathrm{~mm}$ with the Xihui18 value at $8.41 \mathrm{~mm}$ (Fig. S1a and Table1). Chi-square test showed that the ratio of plant number with long grain length (111) to that with short grain length (38) fitted to a 3:1 segregation ratio $\left(\chi^{2}=0.0014<\chi_{0.05,1}^{2}=3.84\right)$. These results suggested that grain length in Z322-1-10 is controlled by a single dominant gene.

\section{QTL mapping for nine agronomic traits}

A total of 13 QTLs were identified in the five substitution segments of Z322-1-10, including one QTL for each of the following traits: plant length, number of spikelets per panicle, grain number per panicle, seed setting ratio, 1000-grains weight and grain length; two QTLs for panicle number per plant and grain width; and three QTLs for the length to width ratio (Fig. 1 and Table 2). QTL $q P L 2$ is located on chromosome 2 linked with marker RM1920. This QTL has an effect of $0.79 \mathrm{~cm}$ and explains $17.28 \%$ of the total

*Further details about the Electronic Supplementary Material (ESM) can be found at the end of the article. 
Table 2. QTLs identified for nine agronomic traits in rice from the $\mathrm{F}_{3}$ population

\begin{tabular}{|l|c|c|c|c|c|c|}
\hline \multicolumn{1}{|c|}{ Traits } & QTL & Chr. & $\begin{array}{c}\text { Linked } \\
\text { marker }\end{array}$ & $\begin{array}{c}\text { Estimated } \\
\text { effect }\end{array}$ & Var\% & P-value \\
\hline Plant length & $q P L 2$ & 2 & RM1920 & 0.79 & 17.28 & 0.015 \\
\hline Panicle number & $q P N 5$ & 5 & RM289 & -0.83 & 7.42 & 0.014 \\
\hline Panicle number & $q P N 6$ & 6 & RM7412 & -0.77 & 6.45 & 0.045 \\
\hline Spikelets per panicle & $q S P P 10$ & 10 & RM7300 & 10.50 & 7.25 & 0.041 \\
\hline Grains per panicle & $q G P P 1$ & 1 & RM3426 & 8.21 & 3.56 & 0.027 \\
\hline Seed setting rate & $q S S R 1$ & 1 & RM3426 & -3.49 & 2.77 & 0.048 \\
\hline Grain weight & $q G W T 1$ & 1 & RM3426 & 0.34 & 2.45 & 0.049 \\
\hline Grain length & $q G L 1$ & 1 & RM3426 & 0.20 & 34.68 & $<.0001$ \\
\hline Grain width & $q G W 5-1$ & 5 & nSSR505 & -0.13 & 44.17 & $<.0001$ \\
\hline Grain width & $q G W 5-2$ & 5 & RM289 & 0.06 & 13.01 & 0.005 \\
\hline Ratio of Length-width & $q R L W 1$ & 1 & RM3426 & 0.05 & 9.27 & 0.002 \\
\hline Ratio of Length-width & $q R L W-5-1$ & 5 & nSSR505 & 0.10 & 33.05 & $<.0001$ \\
\hline Ratio of Length-width & $q R L W 5-2$ & 5 & RM289 & -0.05 & 7.18 & 0.035 \\
\hline
\end{tabular}

phenotypic variance. QTL $q P N 5$, located on chromosome 5 and linked with marker RM289, controls panicle number with an effect of -0.83 that explains $7.42 \%$ of the phenotypic variance. QTL $q P N 6$, located on chromosome 6 and linked with RM7412, controls panicle number with an effect of -0.77 and explaining $6.45 \%$ of the phenotype variance. QTL $q S P P 10$, located on chromosome 10 and linked with RM730, is responsible for spikelets per panicle with an effect of 10.50 at the Nipponbare backgrounds and explaining $7.25 \%$ of phenotypic variance. QTL $q G P P 1$, located on chromosome 1 and linked with RM3426, controls grain number per panicle, can increase grain number to 8.21 per panicle and explain $3.56 \%$ of the phenotypic variance. QTL $q S S R 1$ controls seed setting ratio and is located on the chromosome 1 and linked with RM3426. This QTL explains $2.77 \%$ of the phenotypic variance. Minor effect QTL $q G W T 1$ and major effect QTL $q G L 1$ are both located on the chromosome 1 and linked with RM3426. The minor QTL increases grain weight by $0.34 \mathrm{~g}$ and explains $2.45 \%$ of the phenotypic variance. The major QTL increase grain length by $0.20 \mathrm{~mm}$ and explains $34.68 \%$ of the phenotypic variance. The same substitution segment on chromosome 5 carries two QTLs with effects in opposite direction, where $q G W 5-1$ is linked with nSSR505 and decreases grain width by $0.13 \mathrm{~mm}$ per grain and it explains $44.17 \%$ of the phenotypic variance, $q G W 5-2$ is linked with RM289 and has an effect of 0.06 that explains $13.01 \%$ of phenotypic variance. QTL $q R L W 1$, located on chromosome 1 and linked with RM3426, controls the length to width ratio with an effect of 0.05 and explaining $9.27 \%$ of the phenotypic variance. QTL $q R L W-5-1$, located on the same substitution segment of chromosome 5 and linked with marker nSSR505, controls the length to width ratio with an effect of 0.10 and explaining 
$33.05 \%$ of the phenotypic variance. QTL $q R L W-5-2$, located on the same substitution segment on chromosome 5 and linked with marker RM289, has an effect of -0.05 on the length to width ratio and explain $7.18 \%$ of the phenotypic variance (Table 2 ).

\section{Discussion}

CSSLs are a set of lines developed by a series of backcrosses of a donor parent to a recipient parent until the recipient parent genome is fully recovered except for one or several chromosome segments from the donor parent (Zamir 2001; Ali et al. 2010). Thus, the use of CSSLs can simplify the study of complex genetic traits (Furuta et al. 2014). Once the CSSL libraries are developed in the genetic background of an elite cultivar, the best performing CSSLs may be released as new cultivars. Furthermore, other CSSLs can be used as parents in a breeding program to transfer the introgressed segments with superior performing genes into the elite cultivars by MAS (Ali et al. 2010). CSSLs also provide tools to broaden the genetic variation for plant breeding (Zamir 2001). They can be introgressed from wild species to cultivate relatives (Ali et al. 2010) and from one subspecies to another in rice. CSSLs developed in the background of an indica cultivar with donor segments from japonica varieties. These CSSLs include the one derived from '93-11' (indica recurrent parent) $\times$ Nipponbare (japonica donor) (Wang et al. 2009); Zhenshen 97B (indica recurrent parent) $\times$ Nipponbare (japonica donor) (Zhao et al. 2009); Examples of CSSLs containing donor segments from an indica cultivar in a japonica genetic background include: 'Asominori'(japonica, recurrent parent) $\times$ 'IR24'(indica donor) (Wan et al. 2006); 'Koshihikari' (japonica, recurrent parent) $\times$ 'Nona Bokra'(indica donor) (Takai et al. 2007). In our laboratory, we developed a series of CSSLs derived from Nipponbare (japonica, recurrent) $\times$ Xihui18 (indica, donor parent). Here, a novel elite CSSL (Z322-1-10) was identified. Z322-1-10 carries five chromosome substitution segments with a total length of $24.01 \mathrm{Mb}$ and an average length of $4.80 \mathrm{Mb}$ per segment. These segments are distributed among chromosomes 1, 2, 5, 6 and 10. The line carries desirable agronomic traits, such as larger panicle length, grain length, 1000-grains weight, and more spikelets per panicle than its recipient parent Nipponbare.

Understanding the genetic basis of these quantitative traits is a major challenge. Not only can CSSLs be used as basal materials to verify and characterize QTLs identified, but also can be used for additional backcross that enables us to proceed to fine mapping of QTLs (Ebitani et al. 2005). Here, Z322-1-10 was used to backcross its recurrent parent for mapping of QTLs for nine important agronomic traits. A total of thirteen QTLs were identified. Compared to previous researches, $q G W 5-1$ should be located in the same interval that contains the cloned GW5 between RM3328 and RMw513 (Weng et al. 2008), QTL $q G W 5-2$ was mapped by $\mathrm{Hu}$ et al. (2012). $q L R W 5-1$ was previously mapped in a similar region with the $q R L W 5$ reported by Liu et al. (2015) and $\mathrm{Hu}$ et al. (2012). The $q P N 6$ QTL has also been reported in the same location as ppp6.1 mapped by Cho et al. (2007). qGPP1 might be in the same location as the cloned Gnl (Ashikari et al. 2005). The remaining nine QTLs were not previously reported. These QTLs will all be important for MAS breeding. 
It is worth to mention that there are three major QTLs ( $q G L 1, q G W 5-1$ and $q R L W 5-1)$. Two major QTLs ( $q G W 5-1$ and $q R L W 5-1)$ are closely linked with two minor QTLs ( $q G W 5-2$ and $q R L W 5-2$ ). This leads to a nearly normal distribution of the trait in the $\mathrm{F}_{3}$ population. To dissect the two major QTLs, we need to further decompose the substitution segment on chromosome 5 into two substitution segments by further backcrossing. However, major QTL $q G L 1$ is of a single dominance inheritance, whose frequency distribution for grain length in the $\mathrm{F}_{3}$ population is bimodal, and the ratio long grain to short grain is 3:1. To date, only two major QTLs for grain length (GS3 and GL3.1) on chromosome 3 have been cloned (Fan et al. 2006; Peng et al. 2012). In addition, $q G L 1$ is different from any other previous mapped QTLs such as $q G L 1$ linked with RM1(Liu et al. 2015) and $q g$ l1.1-1.4, qgl2 (Shanmugavadivel et al. 2013), $q G L-2$ (Wan et al. 2006; Liu et al. 2015), qgl3.1-3.2 (Shanmugavadivel et al. 2013), qGL3 (Liu et al. 2015), qgl5.1-5.4, qgl6, qGL-9, qgl11.1-2, qgl12 (Shanmugavadivel et al. 2013; Wan et al. 2006), qGL7 and $q G L 7-2$ (Liu et al. 2015; Wan et al. 2006; Shao et al. 2010). Therefore, the major $q G L 1$ will be important for further cloning and understanding of the molecular mechanisms in regulating yield production.

A novel CSSL that has many favorable agronomic traits for breeding was identified. It carries five chromosome substitution segments, whose average length is $4.80 \mathrm{Mb}$. Using the $\mathrm{F}_{3}$ population derived from the cross of Nipponbare with Z322-1-10, we identified 13 QTLs whose genetic effects explain $2.45 \%$ to $44.17 \%$ of the phenotypic variance of nine agronomic traits. Three major QTLs were found in the substitution segments, of which $q G L 1$ as a single dominant gene and has not been cloned. The study will not only be important for fine mapping and cloning of the gene $G L 1$, but also for pyramiding breeding to incorporate favorable QTL alleles.

\section{Acknowledgements}

The study was supported by "111" project (B12006) and Chongqing key laboratory capacity enhancement project (cstc2014pt-sy80001).

\section{References}

Ali, M.L., Sanchez, P., Yu, S.B., Lorieux, M., Georgia, C., Eizenga, G.C. 2010. Chromosome segment substitution lines: a powerful tool for the introgression of valuable genes from Oryza wild species into cultivated rice (O. sativa). Rice 3:218-234.

Ashikari, M., Sakakibara, H., Lin, S., Yamamoto, T., Takashi, T., Nishimura, A., Angeles, E.R., Qian, Q., Kitano, H., Matsuoka, M. 2005. Cytokinin oxidase regulates rice grain production. Science 309:741-745.

Cho, Y.G., Kang, H.J., Lee, J.S., Lee, Y.T., Lim, S.J., Gauch, H., Eun, M.Y., McCouch, S.R. 2007. Identification of quantitative trait loci in rice for yield, yield components, and agronomic traits across years and locations. Crop Sci. 47:2403-2417.

Ebitani, T., Takeuchi, Y., Nonoue, Y., Yamamoto, T., Takeuchi, K., Yano, M. 2005. Construction and evaluation of chromosome segment substitution lines carrying overlapping chromosome segments of indica rice cultivar Kasalath in a genetic background of japonica elite cultivar Koshihikari. Breed. 55:65-73. 
Fan, C.C., Xing, Y.Z., Mao, H.L., Lu, T.T., Han, B., Xu, C.G., Li, X.H., Zhang, Q.F. 2006. GS3, a major QTL for grain length and weight and minor QTL for grain width and thickness in rice, encodes a putative transmembrane protein. Theor. Appl. Genet. 112:1164-1171.

Furuta, T., Uehara, K., Angeles-Shim, R.B., Shim, J., Ashikari, M., Takashi, T. 2014. Development and evaluation of chromosome segment substitution lines (CSSLs) carrying chromosome segments derived from Oryza rufipogon in the genetic background of Oryza sativa L. Breed. Sci. 63:468-475.

Hu, Z., He, H., Zhang, S., Sun, F., Xin, X., Wang, W., Qian, X., Yang, J., Luo, X. 2012. A kelch motif-containing Serine/Threonine protein phosphatase determines the large grain QTL trait in rice. J. Integr. Plant Biol. 54:979-990.

Liao, C.Y., Wu, P., Hu, B., Yi, K.K. 2001. Effects of genetic background and environment on QTLs and epistasis for rice (Oryza sativa L.) panicle number. Theor. Appl. Genet. 103:104-111.

Liu, D., Kang, M., Wang, F., Liu, W., Fu, C., Li, J., Zhu, M., Zeng, X., Liao, Y., Liu, Z., Huang, H. 2015. Mapping of the genetic determinant for grain size in rice using a recombinant inbred line (RIL) population generated from two elite indica parents. Euphytica 206:159-173.

McCouch, S.R., Kochert, G., Yu, Z.H., Wang, Z,Y., Khush, G.S., Coffman, W.R., Tanksley, S.D. 1988. Molecular mapping of rice chromosomes. Theor. Appl. Genet. 76:148-159.

Panaud, O., Chen, X., McCouch, S.R. 1996. Development of microsatellite markers and characterization of simple sequence length polymorphism in rice (Oryza sativa L). Mol. Genet. Genomics 259:597-607.

Paterson, A.H., Damon, S., Hewitt, J.D., Zamir, D., Rabinowitch, H.D., Lincoln, S.E., Lander, E.S., Tanksley, S.D. 1991. Mendelian factors underlying quantitative traits in tomato: comparison across species, generations, and environments. Genetics 127:181-197.

Peng, Q., Lin, Y.S., Song, X.J., Shen, J.B., Huang, W., Shan, J.X., Zhu, M.Z., Jiang, L., Gao, J.P., Lin, H.X. 2012. The novel quantitative trait locus GL3.1 controls rice grain size and yield by regulating Cyclin-T1;3. Cell Res. 22:1666-1680.

SAS Institute Inc. 2009. SAS/STAT: Users' Guide, Version 9.3. http://suport.sus.com/publishing

Shanmugavadivel, P.S., Amitha-Mithra, S.V., Dokku, P., Anand-Raj-Kumar, K., Rao, G.J.N., Shao, G., Tang, S., Luo, J., Jiao, G., Wei, X., Tang, A., Wu, J., Zhuang, J., Hu, P. 2010. Mapping of qGL7-2, a grain length QTL on chromosome7 of rice. J. Genet. Genomics 37:523-531.

Song, X.J., Huang, W., Min, S., Zhu, M.Z., Lin, H.X. 2007. A QTL for rice grain width and weight encodes a previously unknown RING-type E3 ubiquitin ligase. Nat. Genet. 39:623-630.

Takai, T., Nonoue, Y., Yamamoto, S., Yamanouchi, U., Matsubara, K., Liang, Z.W., Lin, H.X., Ono, N., Uga, Y., Yano, M. 2007. Development of chromosome segment substitution lines derived from backcross between indica donor rice cultivar 'Nona bokra' and japonica recipient cultivar 'koshihikari'. Breeding Sci. 57:257-261.

Wan, X.Y., Wan, J.M., Jiang, L., Wang, J.K., Zhai, H.Q., Weng, J.F., Wang, H.L., Lei, C.L., Wang, J.L., Zhang, X., Cheng, Z.J., Guo, X.P. 2006. QTL analysis for rice grain length and fine mapping of an identified QTL with stable and major effects. Theor. Appl. Genet. 112:1258-1270.

Wang, Y., Sun, Y.J., Chen, D.Y., Yu, S.B. 2009. Analysis of quantitative trait loci in response to nitrogen and phosphorus deficiency in rice using chromosomal segment substitution lines. Acta Agron. Sin. 35:580-587.

Wang, S., Wu, K., Yuan, Q.B., Liu, X., Liu, Z., Liu, X., Zheng, R., Zhu, H., Dong, G., Qian, Q., Zhang, G., Fu, X.D. 2012. Control of grain size, shape and quality by OSSPL16 in rice. Nat. Genet. 44:950-954.

Weng, J.F., Gu. S.H., Wan, X.Y., Gao, H., Guo, T., Su, N., Lei, C.L., Zhang, X., Cheng, Z.J., Guo, X.P., Wang, J.P., Jiang, L., Zhai, H.Q., Wan, J.M. 2008. Isolation and initial characterization of GW5, a major QTL associated with rice grain width and weight. Cell Res. 18:1199-1209.

Xing, Y.Z., Zhang, Q.F. 2010. Genetic and molecular bases of rice yield. Annu. Rev. Plant Biol. 61:11.1-11.22. Yang,Y., Guo, M., Li, R., Shen, L., Wang, W., Liu, M., Zhu, Q., Hu, Z., He, Q., Xue, Y., Tang, S., Gu, M., Yan, C. 2015. Identification of quantitative trait loci responsible for rice grain protein content using chromosome segment substitution lines and fine mapping of $q P C-1$ in rice (Oryza sativa L.) Mol. Breeding 35:130.

Zamir, D. 2001. Improving plant breeding with exotic genetic libraries. Nat. Rev. Genet. 2:983-989.

Zhao, L., Zhou, H., Lu, L., Liu, L., Li, X., Lin, Y., Yu, S. 2009. Identification of quantitative trait loci controlling rice mature seed culturability using chromosomal segment substitution lines. Plant Cell Rep. 28:247256. 
Zheng, T.Q., Wang, Y., Ali, A.J., Zhu, L.H., Sun, Y., Zhai, H.Q, Mei, H.W., Xu, Z.J., Xu, J.L., Li, Z.K. 2011. Genetic effects of background-independent loci for grain weight and shape identified using advanced reciprocal introgression lines from Lemont $\times$ Teqing in rice. Crop Sci. 51:2525-2534.

\section{Electronic Supplementary Material (ESM)}

Electronic Supplementary Material (ESM) associated with this article can be found at the website of CRC at http://www.akademiai.com/content/120427/

Electronic Supplementary Figure S1. Distribution of phenotypic traits in $\mathrm{F}_{3}$ population. N: Nipponbare; Z: Z322-1-10 